ARTICLE

\title{
Oil immersed lossless total analysis system for integrated RNA extraction and detection of SARS- CoV-2
}

\author{
Duane S. Juang ${ }^{1,6}$, Terry D. Juang 1,6 , Dawn M. Dudley², Christina M. Newman², Molly A. Accola ${ }^{3}$, \\ William M. Rehrauer ${ }^{2,3}$, Thomas C. Friedrich (10) ${ }^{4,5}$, David H. O'Connor (1) ${ }^{2,4}$ \& David J. Beebe (1) 1,2凶
}

The COVID-19 pandemic exposed difficulties in scaling current quantitative PCR ( $P P C R$ )based diagnostic methodologies for large-scale infectious disease testing. Bottlenecks include lengthy multi-step processes for nucleic acid extraction followed by qPCR readouts, which require costly instrumentation and infrastructure, as well as reagent and plastic consumable shortages stemming from supply chain constraints. Here we report an Oil Immersed Lossless Total Analysis System (OIL-TAS), which integrates RNA extraction and detection onto a single device that is simple, rapid, cost effective, and requires minimal supplies and infrastructure to perform. We validated the performance of OIL-TAS using contrived SARS-CoV-2 viral particle samples and clinical nasopharyngeal swab samples. OIL-TAS showed a 93\% positive predictive agreement $(n=57)$ and $100 \%$ negative predictive agreement $(n=10)$ with clinical SARS-CoV-2 qPCR assays in testing clinical samples, highlighting its potential to be a faster, cheaper, and easier-to-deploy alternative for infectious disease testing.

\footnotetext{
${ }^{1}$ Department of Biomedical Engineering, University of Wisconsin-Madison, Madison, WI, USA. ${ }^{2}$ Department of Pathology and Laboratory Medicine, University of Wisconsin-Madison, Madison, WI, USA. ${ }^{3}$ UW Health Clinical Laboratories, University of Wisconsin Hospital and Clinics, Madison, WI, USA ${ }^{4}$ Wisconsin National Primate Research Center, University of Wisconsin-Madison, Madison, WI, USA. ${ }^{5}$ Department of Pathobiological Sciences, University of Wisconsin-Madison, Madison, WI, USA. ${ }^{6}$ These authors contributed equally: Duane S. Juang, Terry D. Juang. ${ }^{凶}$ email: djbeebe@wisc.edu
} 
B road testing is crucial for monitoring and controlling the spread of infectious disease outbreaks. The coronavirus disease 2019 (COVID-19) pandemic caused by severe acute respiratory syndrome coronavirus 2 (SARS-CoV-2) spread rapidly around the world after its initial outbreak from Wuhan, China in December 20191,2, quickly overwhelming current diagnostic testing capacity and supply chains. The go-to gold standard diagnostic technique for novel infectious diseases is usually molecular-based (i.e. quantitative polymerase chain reaction (qPCR)), owing to the relative ease to develop a highly sensitive and specific test within a short timeframe. However, existing methodologies for molecular testing have proven difficult to scale, owing to the assay's complexity, lengthy operation, requirement of dedicated centralized testing infrastructure, and supply chain constraints.

The current standard method for SARS-CoV-2 nucleic acid testing is a multi-step protocol involving RNA extraction from patient samples (most commonly nasal, nasopharyngeal or oropharyngeal swabs, and saliva) using magnetic bead-based or column-based methods, followed by reverse transcriptionquantitative PCR (RT-qPCR)-based detection of the extracted RNA. The RNA extraction process typically involves: (1) mixing the sample with lysis/binding buffer, (2) solid-phase capture of the viral RNA via magnetic beads or columns, (3) multiple washes involving magnetic separation or centrifugation for each wash, (4) elution of viral RNA with water or a low salt buffer, (5) adding the eluted RNA to a PCR plate containing RT-qPCR master mix and primers, followed by thermocycling and data capture in a qPCR machine. This process often takes up to $4 \mathrm{~h}$ and is challenging to scale because of the complexity of the RNA extraction process, coupled with the RT-qPCR process itself which requires over $1 \mathrm{~h}$ of on-machine real-time fluorescence measurements, significantly limiting assay turnaround time. The nature of this complex multi-step process also necessitates the use of a significant amount of plastic consumables (pipet tips, tubes, plates, columns, etc.) which become biohazardous waste after the assay. For example, it is estimated that up to 10 pipet tips (6 for RNA extraction, 4 for PCR setup) are consumed per sample for a SARS-CoV-2 qPCR-based molecular test ${ }^{3}$. This has also led to widespread shortages of essential plastic consumables to perform the tests, significantly bottlenecking testing capacity ${ }^{4}$.

To address these challenges for scaling up testing, here we report an alternative molecular assay we termed the oil immersed lossless total analysis system (OIL-TAS) which integrates RNA extraction and detection into a single device with the footprint of a generic 96-well plate. The OIL-TAS is an integration of three main technologies: (1) an underoil droplet microfluidic technology called exclusive liquid repellency (ELR) that allows for lossless sample processing 5,$6 ;(2)$ a rapid solid-phase analyte extraction method called exclusion-based sample preparation (ESP) ${ }^{7-12}$; and (3) isothermal amplification with colorimetric readout (loopmediated isothermal amplification, LAMP ${ }^{13,14}$ ). The OIL-TAS has advantages over traditional RT-qPCR assays including simplicity, lossless sample handling, less reagent/plastic consumable consumption, lower cost, and increased speed and throughput. Importantly, the OIL-TAS can be operated using pipettes, a shaker, an oven, and an image capture device, which are widely available in biomedical laboratories without the need for costly or specialized instruments. We also deliberately engineered the assay to be compatible with open-source, non-proprietary reagents, and employ a colorimetric isothermal detection method to reduce assay time and avoid adding burden to current qPCR testing supply chains and clinical workflows. We demonstrate that the OIL-TAS can reliably detect a concentration of 10 copies $/ \mu \mathrm{L}$ and sporadically detect down to 1 copy/ $\mu \mathrm{L}$ with contrived SARS-CoV2 viral particle samples. When testing clinical nasopharyngeal swab samples, OIL-TAS showed a $93 \%$ positive predictive agreement $(n=57)$ and $100 \%$ negative predictive agreement $(n=10)$ with clinical SARS-CoV-2 qPCR assays.

\section{Results}

Operation principle and design of the OIL-TAS. The OIL-TAS builds upon our previously reported ELR technology, which describes physical conditions where an aqueous droplet can be fully repelled from a solid surface (contact angle $=180^{\circ}$ ) in the presence of an oil phase when a specific set of oil and solid interfacial energy properties are met. The conditions in which ELR will occur are when the sum of the interfacial energies of the solid/oil and aqueous/oil interface is equal to, or less than the solid/aqueous interfacial energy. Through experimentation, we found that this condition can be accomplished by employing a polydimethylsiloxane (PDMS)-silane functionalized surface paired with silicone oil as the oil phase ${ }^{5}$. The most significant advantage of ELR is that it prevents adsorption of biological samples to surfaces and thus has very little, if any, associated sample loss. With ELR, one can create individually isolated aqueous droplets immersed under a common oil phase, each droplet providing a completely isolated reaction condition with no crosstalk with the solid surface of the reservoir, enabling liquid handling without loss.

Although ELR can effectively mitigate surface adsorptionmediated sample loss, another common source of loss during sample processing often occurs during solid-phase extraction processes: namely, target analytes (such as RNA) that are bound to a solid phase (magnetic beads or column resin) can fall off prematurely during the multiple washing steps that are common in solid-phase extraction processes such as RNA extraction. This issue can be effectively reduced by employing ESP, which refers to a collection of solid-phase analyte extraction techniques by which analytes bound to a solid-phase (magnetic beads) can be extracted out of a complex sample by transporting the beads via a magnet through an immiscible interface (oil or air) to "exclude" nontarget contaminants from the sample. The ESP process replaces the multiple washing operations in traditional solid-phase extraction techniques with a simple magnetic dragging operation through the immiscible interfaces, resulting in a much shorter processing time and higher sample recovery ${ }^{7-12,15-17}$.

OIL-TAS combines the ELR and ESP technologies into a rapid and flexible integrated analyte extraction and detection platform. The OIL-TAS consists of an array of immobilized ELR droplets immersed under an oil bath, where each droplet can contain a sample, wash solution, or reaction solution (Fig. 1). The oil phase provides droplet isolation and liquid repellent properties, and also serves as a water-immiscible extraction interface for performing ESP: magnetic beads can be added to the aqueous droplets and "extracted" from one droplet to another by dragging a magnet across the bottom of the device for analyte purification (Fig. 1 and Supplementary Movie 1). Additionally, the oil overlay also provides many other advantages including: (1) it prevents evaporation of droplets, thus enabling reactions that require heating (such as isothermal amplification) to be performed using much smaller volumes, (2) it prevents cross-contamination of reagents/samples and LAMP-amplified products, (3) it prevents aerosol formation during operation, as aerosols would be effectively trapped under the oil overlay, (4) it prevents contamination from the environment, as dust particles/other contaminants would be shielded by the oil overlay, and (5) it enables longer-term storage of individual aqueous reagents in the plate via freezing $\left(-20^{\circ} \mathrm{C}\right)$.

In order to robustly immobilize the spherical ELR droplets, we designed an array of wells for trapping the droplets, with shallow extraction channels interconnecting the wells for performing extraction (Fig. 1). One extraction unit of the device consists of a large sample well connected to three small wells (for wash 1, wash 


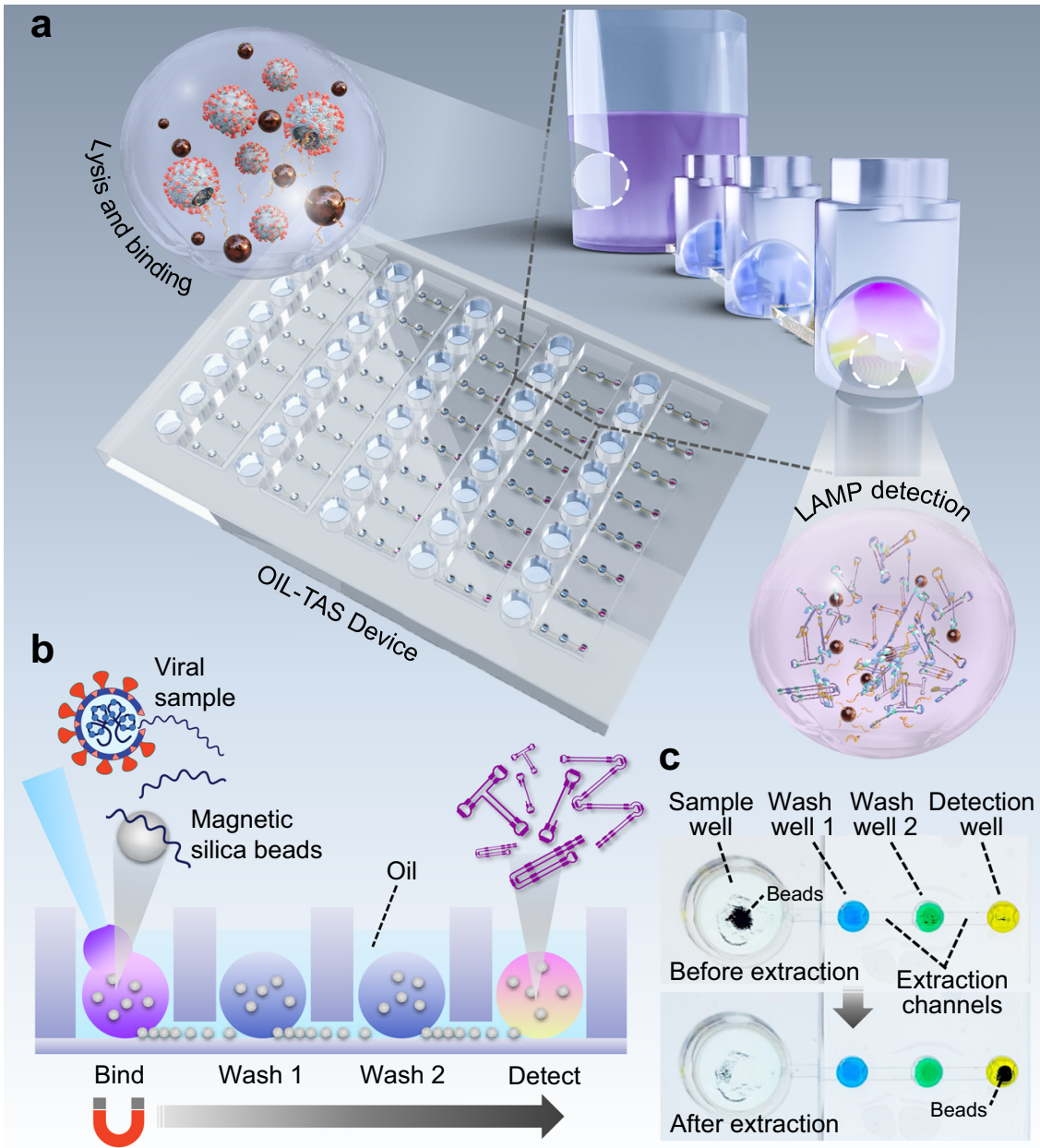

Fig. 1 Integrated RNA extraction and detection with the OIL-TAS assay. a Schematic depicting the design and essential components of the OIL-TAS. b Side view cartoon illustrating the operation principle of OIL-TAS sample extraction. Owing to the exclusive liquid-repellent (ELR) nature of the device surface, droplets appear spherical which minimizes contact with the device surface and hence biofouling-associated sample loss. c Images of a single unit of the OIL-TAS device before and after extraction. Each unit includes a large sample well for sample lysis and binding, followed by two washing wells and a detection well. Interconnecting the wells are oil-filled extraction channels. Wells are filled with droplets of food coloring for visualization.

2, and detection) (Fig. 1b, c). Each device is the size of a microtiter plate $(127.76 \mathrm{~mm} \times 85.48 \mathrm{~mm})$ and contains 40 extraction units which allow for the simultaneous parallel processing of 40 samples. The whole device is treated using ELR chemistry, which ensures that any given surface of the device is fully repellent to aqueous media to prevent biomolecule adsorption. Adding silicone oil into the sample well fills all downstream wells and extraction channels concurrently with oil, owing to hydrophobic wetting from the ELR surface coating. When aqueous droplets are pipetted into the oilfilled wells, it forms an array of oil-immersed aqueous droplets separated by oil-filled extraction channels (Fig. 1). The hydrophobicity of the ELR coating also prevents the aqueous droplets from wicking into the extraction channels. The device is fabricated from three sheets of heat-resistant, optically transparent plastic (polycarbonate, Lexan) which provides advantages including a wide working temperature range $\left(-40\right.$ to $\left.115^{\circ} \mathrm{C}\right)$, uniform chemical properties throughout the device, and compatibility with mass manufacturing injection molding processes (Fig. 2a). The optically clear thin device bottom $(127 \mu \mathrm{m})$ allows for efficient magnetic manipulation, rapid heat transfer, and optical access, making the device compatible with visual, absorbance, fluorescence, luminescence, and microscopy-based readouts. The vertical pitch of the wells is $9 \mathrm{~mm}$ to enable parallel operation via a standard multichannel pipet, whereas the horizontal pitch is
$4.5 \mathrm{~mm}$ (corresponding to a conventional 384-well plate) to enable data acquisition via microplate readers. The inlets of the small wells (wash well 1 , wash well 2 , and detection well) incorporate a non-circular collar to help align the pipet tip during liquid dispensing (Fig. 2b). The non-circular collar design can prevent the circular pipet tip from forming a tight seal over the inlet, allowing oil to escape over the top of the well when being displaced by pipetting aqueous media into the well. The collar also prevents the aqueous droplet from accidental escape from the top of the well, as long as the diameter of the dispensed droplet is larger than the opening of the collar.

Assay design and characterization. When using the OIL-TAS device for SARS-CoV-2 testing, the number of operation steps is minimized to (1) add oil, washing solutions, and LAMP reaction solution into the device, (2) mix sample and lysis buffer/beads in a plate, (3) transfer the bead/sample mixture to the device via a pipet, and perform extraction by dragging a magnet across the bottom of the device, (4) place the device in an oven for isothermal amplification, and (5) data readout via visual inspection or an image acquisition device (flatbed scanner, camera/smartphone, or plate reader) (Supplementary Fig. 1). An operator can perform the whole process manually in $<30$ min for a 40 -sample device (excluding the 


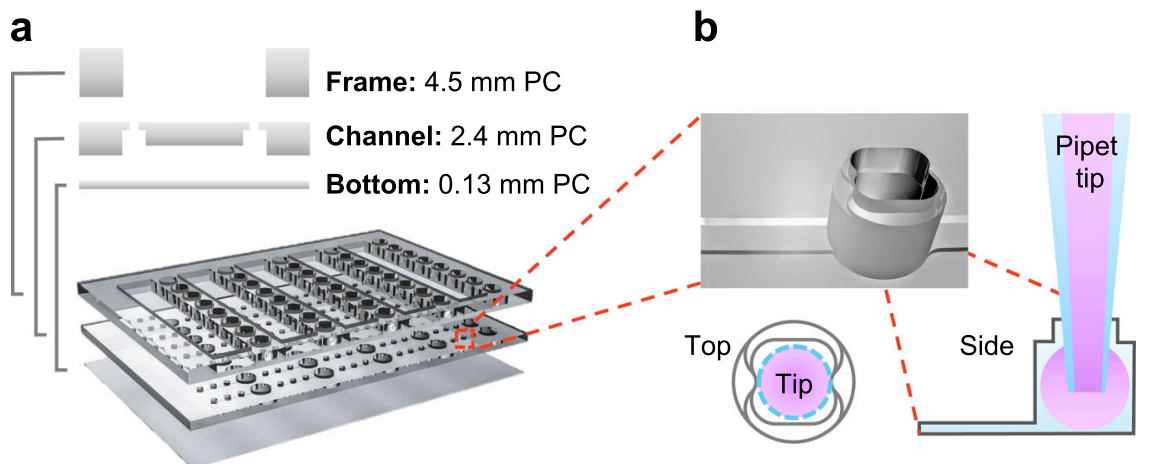

C

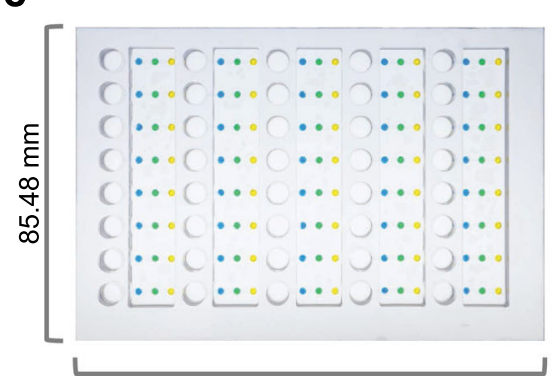

$127.76 \mathrm{~mm}$

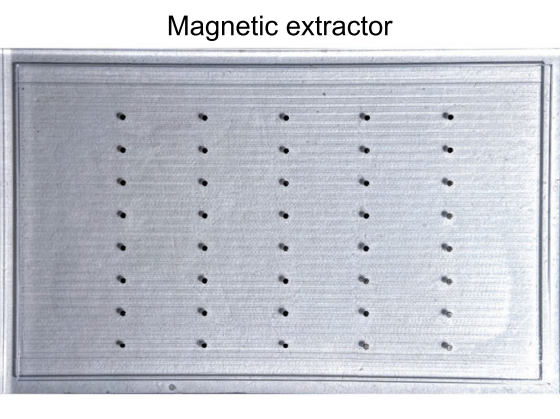

Fig. 2 Design and assembly of the OIL-TAS device. a Exploded view of the OIL-TAS device consisting of three layers fabricated from polycarbonate (PC). b Design of well collar for pipet tip alignment and preventing droplet escape. c Images of the OIL-TAS device (left) and magnetic extractor (right). Food coloring was added to the wells to facilitate visualization.

isothermal amplification time), although this can be significantly shortened by using reagent pre-loaded plates and an automated liquid handling system. This compares favorably to commercial automated RNA extraction systems, which commonly take between 45 and 120 min to process 96 samples ${ }^{18-20}$. It is worth noting that the reason for performing the sample lysis/bead binding step offdevice on a well plate is to enable bead mixing via orbital shaker, as well plates are available with taller walls to prevent spillage and cross-well contamination during shaking. Fabricating the OIL-TAS device with comparably tall walls to enable on-device bead mixing is feasible with injection molding processes but is more challenging and impractical to manufacture at scale using lab prototyping techniques (CNC milling).

To quantify the sample purification performance of the OILTAS method, we evaluated the amount of liquid carryover from one droplet to another during the magnetic extraction process in the OIL-TAS device using a fluorescent molecule (acridine orange) as a model contaminant (Supplementary Fig. 2). Results show only $46.4 \mathrm{~nL}$ of the aqueous solution was carried over from one droplet to another during the magnetic extraction process (equivalent to $1.16 \%$ of the $4 \mu \mathrm{L}$ droplet), which means that $\sim 0.00015 \%$ of contaminating solution will remain after the three sequential extractions (Supplementary Fig. 2). Owing to the harsh protein denaturing properties of the lysis/binding buffer, we implemented two washes as a fail-safe measure to prevent any residual carryover from inhibiting the downstream LAMP reaction. We also tested a variety of channel heights ranging between $100-600 \mu \mathrm{m}$ and found that magnetic extraction works throughout this broad range of channel heights (Supplementary Movie 2). However, to prevent bead clogging during extraction or accidental droplet escape through the extraction channels during device handling, we selected an optimized height of $200 \mu \mathrm{m}$ and a width of $800 \mu \mathrm{m}$ for the extraction channels. We validated that the droplets in this design were highly stable without any observed escape or cross-contamination after rocking on a rocking platform shaker at $30 \mathrm{rpm}$ for $30 \mathrm{~min}$ (Supplementary Fig. 3 and Supplementary Movie 3) or even after vigorous shaking on an orbital plate shaker at $900 \mathrm{rpm}$ (Supplementary Movie 3).

To avoid the shortage and supply chain issues with scaling up testing for SARS-CoV-2, we designed our assay to (1) be compatible with completely open-source, non-proprietary materials and reagents for RNA extraction, and (2) use very little $(4 \mu \mathrm{L})$ proprietary reagent (colorimetric LAMP master mix) per reaction to conserve on limited materials and reduce cost. The main bottleneck in RNA extraction supplies is the proprietary nature of most reagents in commercial RNA extraction kits, including the lysis/binding buffer, washing buffers, as well as magnetic beads, which constrains supply to the production capacity of a given company. However, it is known that the majority of commercial solid-phase RNA extraction methods employ variants of a guanidine salt/silica binding chemistry reported in 1990 by Boom et al. ${ }^{21}$. RNA binds to silica surfaces (such as silica columns or magnetic silica beads) under high guanidine salt conditions in the presence of an alcohol (commonly ethanol or isopropanol), which are the major active ingredients in RNA lysis/binding buffers. There have been efforts to develop various open-source RNA lysis/binding buffer recipes from the scientific community ${ }^{22-26}$, which were demonstrated by the authors to be similar in performance to commercial proprietary kits. We adopted a lysis buffer recipe reported by Escobar et al. ${ }^{22}$ with slight modifications (recipe in the "Methods" section) and found it to perform well in our system. We also tested magnetic silica beads from different vendors (MagAttract beads from Qiagen, MagneSil beads from Promega, MagBinding beads from Zymo Research, and SeraSil-Mag beads from Cytiva) for compatibility with this open-source RNA extraction chemistry and our OIL-TAS system. We found that the beads from these vendors were all compatible with the OIL-TAS extraction process and successfully extracted RNA from gamma-irradiated inactivated SARS-CoV-2 viral particles spiked into viral transport media (VTM) to yield good sensitivity via LAMP detection with an $N$ gene 


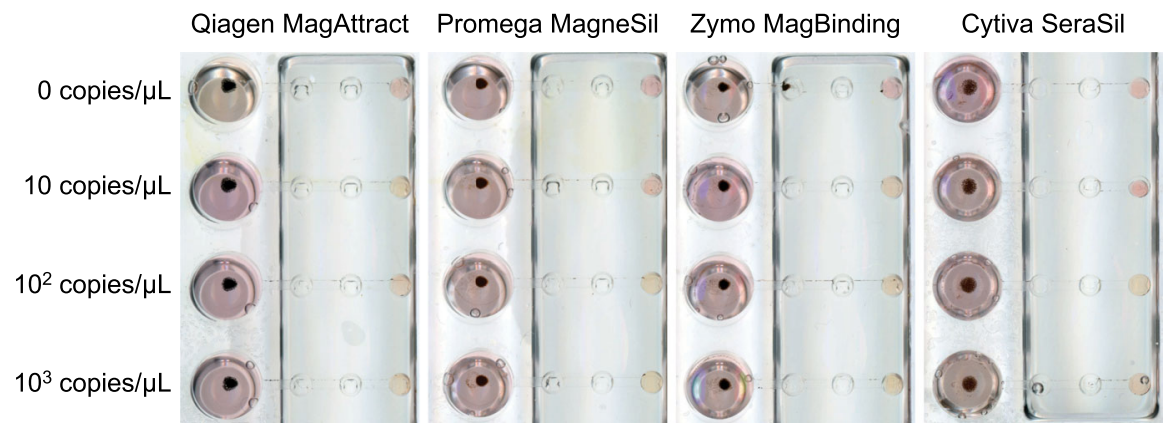

Fig. 3 Integrated extraction and LAMP detection of $\boldsymbol{\gamma}$-irradiated inactivated SARS-CoV-2 particles with OIL-TAS. Performance of the OIL-TAS assay with magnetic silica beads from various vendors paired with an open-source lysis buffer (recipe in the "Methods" section) for integrated RNA extraction and LAMP detection of SARS-CoV-2. Magnetic beads are magnetically moved back into the sample (large) well following LAMP amplification to enable clearer visualization of color change.

a

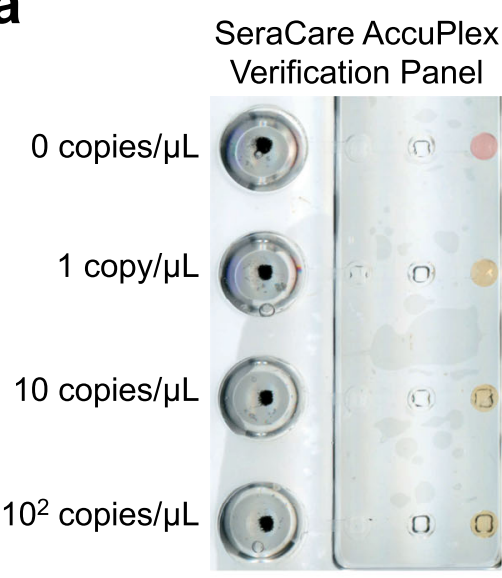

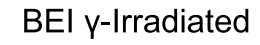
SARS-CoV-2

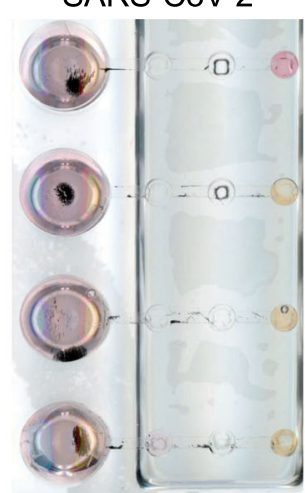

C

\begin{tabular}{|c|c|}
\hline Concentration & Detection rate (\%) \\
\hline 0 copies $/ \mu \mathrm{L}$ & $0 / 10(0 \%)$ \\
\hline $1 \mathrm{copy} / \mu \mathrm{L}$ & $6 / 10(60 \%)$ \\
\hline 10 copies $/ \mu \mathrm{L}$ & $10 / 10(100 \%)$ \\
\hline $10^{2}$ copies $/ \mu \mathrm{L}$ & $10 / 10(100 \%)$ \\
\hline
\end{tabular}

b

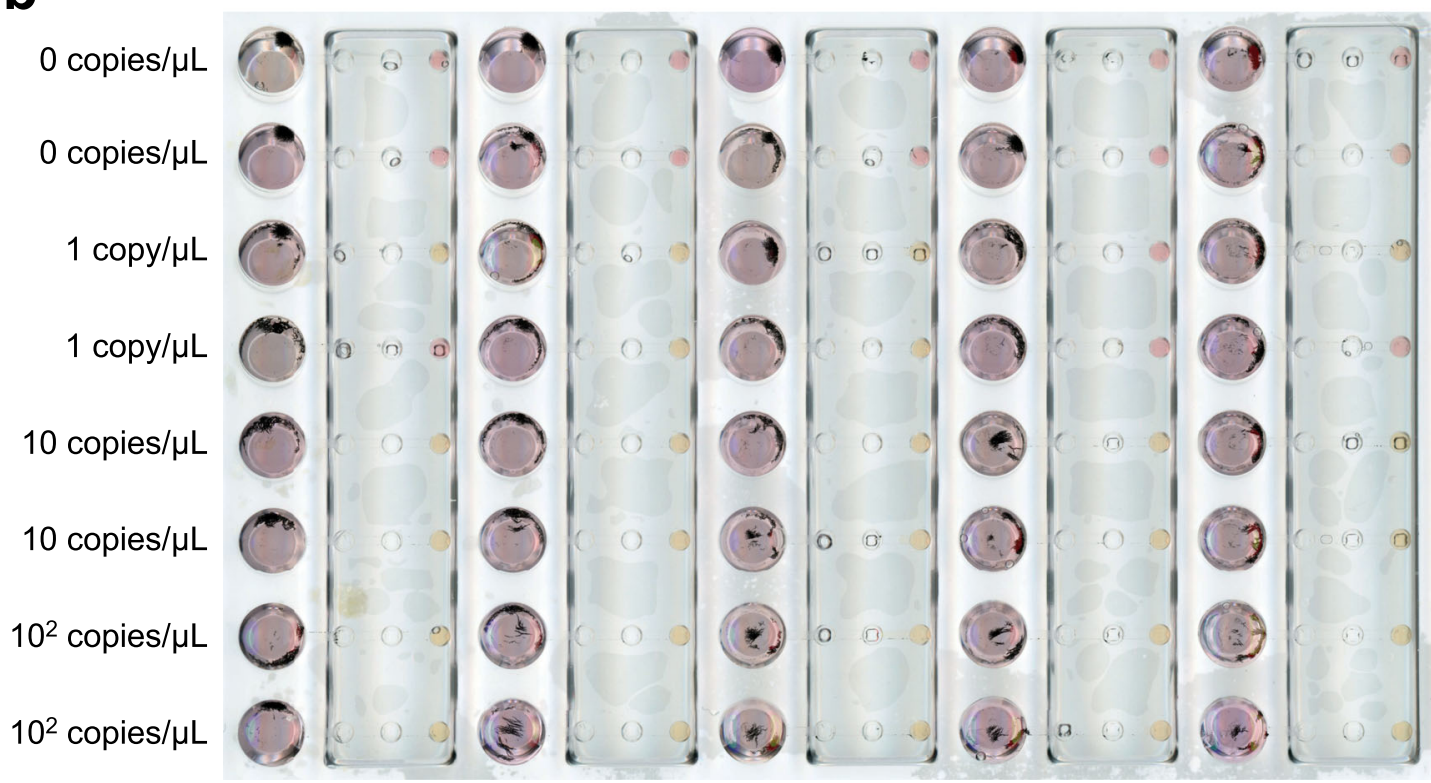

Fig. 4 Extraction and detection of contrived SARS-CoV-2 viral particle samples using OIL-TAS. a RNA extraction and detection of viral particles. Left, synthetic viral particles containing SARS-CoV-2 consensus sequences (AccuPlex verification panel, SeraCare Life Sciences Inc.). Right, $\gamma$-irradiated inactivated SARS-CoV-2 particles (strain USA-WA1/2020) from BEI. b Sensitivity of the OIL-TAS assay for detecting SARS-CoV-2 viral particles across 10 replicates. c Detection rate of the OIL-TAS assay across different viral particle concentrations (quantified from panel $\mathbf{b}$ ). 
a

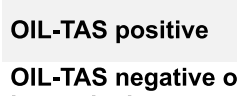
inconclusive

Agreement
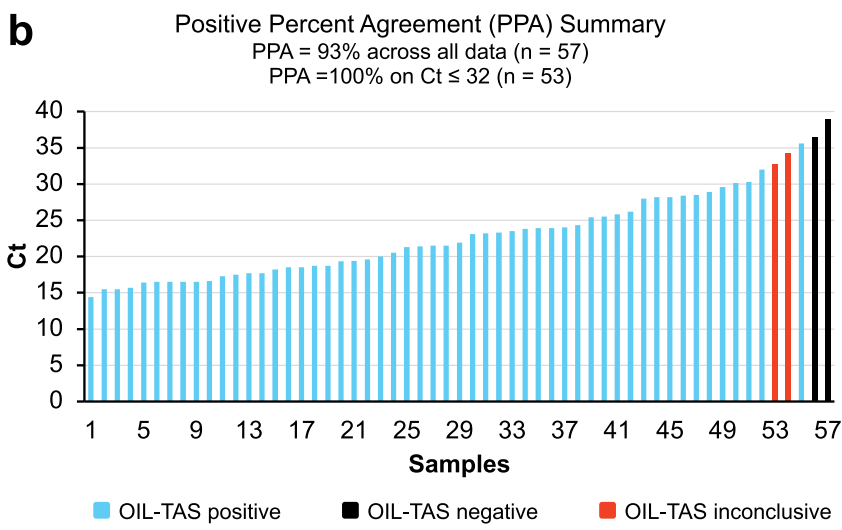

Fig. 5 Clinical sample assay performance of OIL-TAS compared to RTqPCR. a A total of 67 clinical NP swab samples (57 positive and 10 negative for SARS-CoV-2 determined by RT-qPCR) were tested using the OIL-TAS assay $(n=3)$. PPA positive percent agreement, NPA negative percent agreement. b Summary of PPA with RT-qPCR Ct values across 57 SARS-CoV2 positive samples. Out of the 57 PCR-positive samples, 53 tested positive with OIL-TAS ( $3 / 3$ detected), 2 samples tested negative ( $0 / 3$ detected), and 2 samples were inconclusive ( $1 / 3$ detected). RT-qPCR Ct values are plotted in increasing order. Source data are provided as a Source Data file.

primer set $^{27}$ targeting SARS-CoV-2 (Fig. 3). We selected the $N$ Gene primer set reported by Broughton et al. ${ }^{27}$ because it was granted emergency use authorization (EUA) by the U.S. Food and Drug Administration for Mammoth Biosciences ${ }^{28}$ and Color Genomics $^{29}$ for SARS-CoV-2 testing. We did observe minor differences in magnetic responsiveness and performance between the beads from different vendors, although minor optimizations for each bead type may further improve performance. Qiagen MagAttract beads were selected for the following experiments due to their higher magnetic responsiveness and sensitivity.

To further improve on-site assay throughput and operation simplicity, we also tried preloading the OIL-TAS device to contain most of the necessary assay reagents (oil, washing solutions, and LAMP master mix) followed by freezer storage $\left(-20^{\circ} \mathrm{C}\right)$. We found that after a week-long storage at $-20^{\circ} \mathrm{C}$ (longer periods were not tested), the OIL-TAS device still retained similar performance after the freeze-thaw compared to a freshly prepared device (Supplementary Fig. 4), suggesting the potential for pre-packaging assays into a ready-to-run format in OIL-TAS.

Performance of the OIL-TAS assay in detecting SARS-CoV-2. We further validated the performance and sensitivity of the assay using replication-deficient viral particle samples (SeraCare AccuPlex SARS-CoV-2 Verification Panel) in viral transport media. Results show that we were able to detect down to 1 copy/ $\mu \mathrm{L}$ (Fig. $4 \mathrm{a}$, left panel) using the $N$ Gene primer set. The assay also showed no false positives using the RNase $\mathrm{P}$ negative extraction control samples included in the SeraCare AccuPlex kit. We observed similar sensitivity of the assay using BEI gamma-irradiated inactivated SARSCoV-2 viral particles spiked into VTM (Fig. 4a, right panel). The analytical limit of detection (LoD) of the assay for SARS-CoV-2 viral particles was evaluated by three serial 10 -fold dilutions of gamma- irradiated viral particles with 10 replicates. Results show that we were able to detect $6 / 10$ samples for 1 copy/ $\mu \mathrm{L}$, and $10 / 10$ for 10 and 100 copies $/ \mu \mathrm{L}$ concentrations (Fig. $4 \mathrm{~b}, \mathrm{c}$ ), with none detected $(0 / 10)$ for 0 copies $/ \mu \mathrm{L}$. These results show that our assay can reliably detect down to 10 copies $/ \mu \mathrm{L}$ and approach 1 copy/ $\mu \mathrm{L}$ sensitivity, with good specificity.

We next tested the performance of OIL-TAS in assaying nasopharyngeal (NP) swab clinical samples from 57 SARS-CoV-2 PCR-positive and 10 SARS-CoV-2 PCR-negative patients stored in four different transport media (universal transport media (UTM), room temperature M4 (RT M4), phosphate-buffered saline (PBS) and saline). The positive samples comprise a wide range of threshold cycle $(\mathrm{Ct})$ values ranging from 14.4 to 39 . Results show that OILTAS was able to successfully assay NP swab samples across all 4 transport media with $93 \%$ positive percent agreement (PPA) $(53 / 57$, $n=3)$ and $100 \%$ negative percent agreement (NPA) $(10 / 10, n=3)$ with RT-qPCR (Fig. 5a, Supplementary Table 1). Out of the 4 discrepant PCR-positive samples, 2 were inconclusive (1/3 detected, RT-qPCR Ct $=34.22$ and 32.7, respectively), and 2 were negative ( $0 /$ 3 detected, RT-qPCR $\mathrm{Ct}=39$ and 36.5, respectively) (Fig. 5b, Supplementary Table 1). Worth noting is that all clinical samples were heat inactivated at $65^{\circ} \mathrm{C}$ for $30 \mathrm{~min}$ (to enable safe handling at biosafety level 2) and underwent a freeze-thaw cycle (stored at $-80^{\circ}$ $\mathrm{C}$ then thawed at room temperature) prior to running the OIL-TAS assay, which may have a minor impact on sensitivity. On the other hand, the RT-qPCR clinical assays were directly performed on samples without heat inactivation or freeze-thawing.

Multiplex detection with the OIL-TAS assay. Most nucleic acidbased diagnostic assays include an extraction (process) control readout in addition to the target sequence to validate that the assay is performing correctly. Thus, we designed a single input, dual output version of the OIL-TAS device (Fig. 6a) to enable simultaneous multiplexed readout from a single sample to increase the robustness of the assay. The dual output device footprint remains the same and also has the same number of input sample wells (40 samples) as the single output device, but the number of small wells (wash wells and detection wells) was doubled (Fig. 6b). This was accomplished by halving the horizontal pitch between the small wells to $4.5 \mathrm{~mm}$, corresponding to that of a 384-well plate, and increasing the size of the sample wells to accommodate larger sample volumes. We employed this design for the simultaneous detection of the $N$ gene from SARS-CoV-2 and human RNase $\mathrm{P}$ as an extraction process control or the simultaneous detection of two genomic targets from SARS-CoV-2 ( $N$ gene and ORF1a). This was done by using different primer sets in the 2 output wells, including primers for the $N$ gene targeting SARS-CoV- $2^{27}$, Asle targeting the open-reading frame (ORF1a) of SARS-CoV-2 ${ }^{30}$, and RNase P targeting human cells as a process control $^{31}$. We spiked human A549 cells at a concentration of 10 cells $/ \mu \mathrm{L}$ into VTM containing $0,1,10$, and 100 copies $/ \mu \mathrm{L}$ inactivated SARS-CoV-2 particles as a contrived sample. Results show that the sensitivity of the assay was similar with the dual output device, detecting down to 1 copy/ $\mu \mathrm{L}$ SARS-CoV-2 viral particles, with all the RNase $\mathrm{P}$ extraction controls positive (Fig. 6c). Multiplexed detection of SARS-CoV-2 using two primers also show similar sensitivity (Fig. 6d). Worth mentioning is that at 1 copy/ $\mu \mathrm{L}$ concentrations, our detection rate was $6 / 10$ using the single output device (Fig. 4c), but the sensitivity and reliability can be potentially improved in the dual output device as 1 of the 2 primer sets can still detect a positive signal (Fig. 6d). Multiplexed detection of the $N$ gene and RNase P on clinical NP swab samples 1-8 using the dual-output device showed consistent results with the single output device (Fig. 6e, Supplementary Table 1). Simultaneous dual extraction of an RNase P sample also 

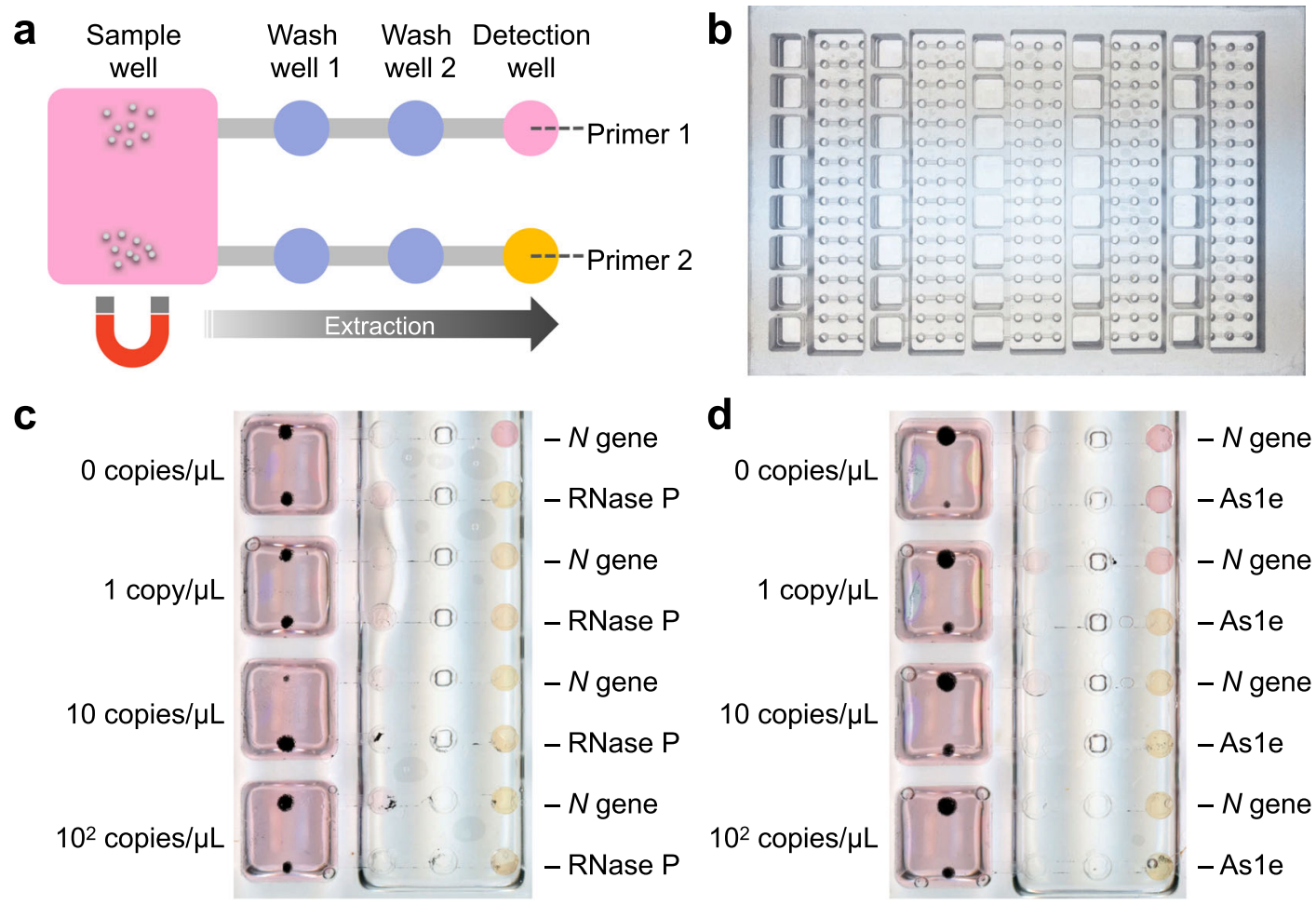

e

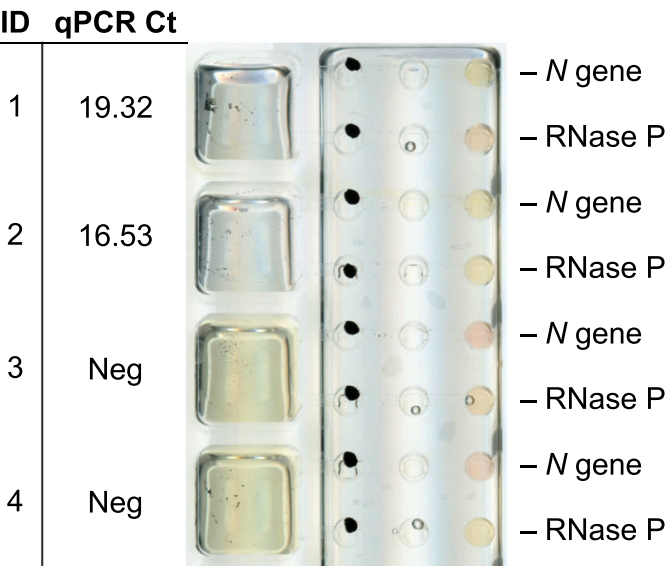

\section{ID qPCR Ct}

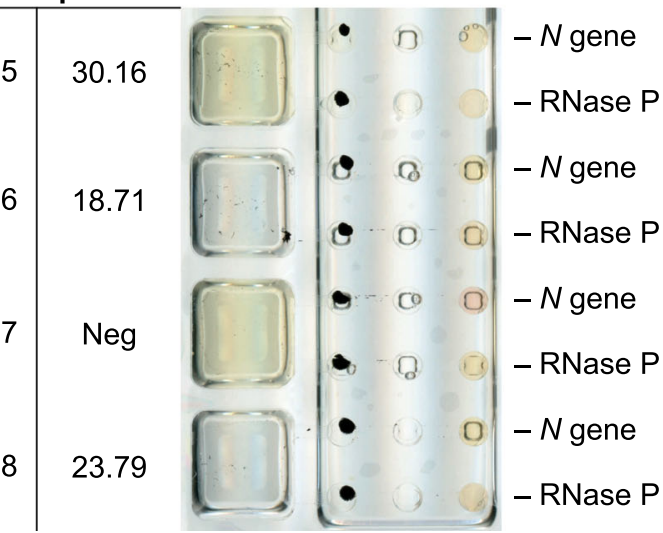

Fig. 6 Dual output OIL-TAS device enables simultaneous multiplexed detection from a single sample. a OIL-TAS can be designed with multiple extraction outputs (two outputs shown here) leading from a single input sample well, enabling multiplexed detection of a sample by using different primers in the detection well. $\mathbf{b}$ Image of the dual-output OIL-TAS device which has the same footprint as a 96 -well plate $(127.76 \mathrm{~mm} \times 85.48 \mathrm{~mm})$. c Multiplexed detection of the SARS-CoV-2 N gene with an extraction positive control (RNase P). $\gamma$-irradiated inactivated SARS-CoV-2 particles were spiked into a cell suspension containing 10 A549 cells/ $\mu \mathrm{L}$ in VTM to mimic a clinical sample. d Multiplexed detection of two different regions of the SARS-CoV-2 genome (As1e, which targets the open-reading frame (ORF1a), and the $N$ gene). e Multiplexed detection of the $N$ gene and RNase P on clinical NP swab samples 1-8 (from Supplementary Table 1).

showed that the two outputs have equivalent extraction performance as indicated by time to threshold values of LAMP RNase P amplification (Supplementary Fig. 5). Worth noting is that the number of output wells (and hence multiplexing targets) may be scaled up with just minor modifications to the current design.

\section{Discussion}

In summary, the OIL-TAS method provides a simple and fast integrated solution from sample to answer for SARS-CoV-2 testing. The OIL-TAS is advantageous in several ways: (1) greatly reduced operation steps. RNA extraction and data acquisition is performed on the same device; (2) reduction in biohazardous medical waste; (3) extraction process is done under an oil overlay, preventing cross- contamination and aerosol formation; (4) endpoint readout decouples reaction (isothermal amplification at $65^{\circ} \mathrm{C}$ in an oven) from detection (flatbed scanner), thus freeing up instrument time for increased assay turnaround. A single operator can process a device (40 samples) within $30 \mathrm{~min}$ manually, which, including the $35 \mathrm{~min}$ incubation time adds up to a sample-to-result time of $~ 70 \mathrm{~min}$; (5) very low assay reagent and instrumentation cost; and (6) high specificity and good sensitivity. Importantly, although here we demonstrated the OIL-TAS using a manual operation protocol, the conventional plate format of the OIL-TAS device makes it very easily adaptable to an automated workflow using commercially available automated liquid handling systems and plate readers for further increased throughput. We have also shown that the OIL-TAS assay 
has good sensitivity for a LAMP-based test and was able to detect all SARS-CoV-2 positive NP swab samples with a $\mathrm{qPCR} \mathrm{Ct} \leq 32$. However, we acknowledge that further validation and potential assay modifications would be required for using the OIL-TAS assay on different sample types (i.e., oropharyngeal swabs, saliva, etc.). Worth noting is that the thermoresistant construction of the OIL-TAS device makes it compatible with thermocycling operations, potentially enabling alternative readouts such as qPCR. We also envision that the modular OIL-TAS design can be scaled down to a single patient, point-of-care diagnostic device for on-site testing needs.

\section{Methods}

Cell culture. A549 human lung cancer cells were sourced from ATCC.

Device fabrication. Devices were milled out of $2.4 \mathrm{~mm}$ (for channels) and $4.5 \mathrm{~mm}$ (for top frame) thick polycarbonate sheets (LEXAN 9034, United States Plastic Corporation) using a computer numerical control (CNC) three-axis mill (Tormach, PCNC 770 ). Following milling, the two pieces were thoroughly washed with $100 \%$ isopropyl alcohol and air dried using an air gun. The two milled pieces were then aligned and placed on top of a 0.005 inch $(127 \mu \mathrm{m})$ thick polycarbonate sheet (TAP Plastics) and bonded together in a thermal press (Carver Press, 3889.1NE1001) with a pressure of $3000 \mathrm{~kg}$ for $40 \mathrm{~min}$. The bonded device was then treated with oxygen plasma for $2 \mathrm{~min}$ at $100 \mathrm{~W}$ (Diener Electronic Femto, Plasma Surface Technology). After plasma treatment, the device was placed in a vacuum desiccator with two trays $(40 \mu \mathrm{L}$ each) of PDMS silane (1,3'dichlorotetramethylsiloxane, Gelest, SID3372.0). The desiccator was then pumped down to vaporize and condense the PDMS silane onto the device surface at RT overnight to functionalize the device surface. The device was then thoroughly rinsed with $100 \%$ isopropyl alcohol then dried using an air gun to remove residual unattached PDMS silane.

Reagent and sample preparation. Colorimetric LAMP master mix was prepared by mixing $100 \mu \mathrm{L}$ of WarmStart Colorimetric LAMP 2X Master Mix (New England Biolabs), $20 \mu \mathrm{L}$ of 10X LAMP primer mix, and $80 \mu \mathrm{L}$ of nuclease-free water (for $1 \mathrm{OIL}-$ TAS device). LAMP primer sets were purchased from Integrated DNA Technologies, with primer sequences shown in Supplementary Table 2. The primers used include an $N$ Gene primer ${ }^{27}$, Asle primer ${ }^{30}$, and RNase $\mathrm{P}$ primer $^{31}$. The RNA lysis buffer was prepared using $4 \mathrm{M}$ guanidine thiocyanate, $10 \mathrm{mM}$ MES (2-ethanesulfonic acid), $1 \%$ Triton X-100, with 1\% B-mercaptoethanol added right before use. Qiagen MagAttract magnetic silica beads were diluted in $99 \%$ ethanol to reach an equivalent of $0.25 \mu \mathrm{L}$ bead stock/extraction. For bead washing, 50\% ethanol and nuclease-free water was used for wash 1 and wash 2, respectively. Worth noting is that the rapid $(\sim 1 \mathrm{~s})$ and gentle nature of "washing" in the OIL-TAS method (dragging beads through a droplet) allowed us to use water as a washing solution while retaining good sensitivity. Gammairradiated inactivated SARS-CoV-2 viral particles (strain USA-WA1/2020) were obtained from the Biodefense and Emerging Infections Research Resources Repository (BEI), (cat\# NR-52287) and serially diluted in VTM.

Device operation (single output device). To prepare the OIL-TAS device, $30 \mu \mathrm{L}$ of silicone oil was added to each of the sample wells, which, due to the nature of the ELR surface treatment, the oil simultaneously wicks into the extraction channels and wells, forming an oil barrier to separate each well and also prevents sample evaporation. Using a p20 pipette, $4 \mu \mathrm{L}$ of nuclease-free water, $50 \%$ ethanol, and LAMP master mix were sequentially added to washing well 2 , washing well 1 , and the detection well, respectively. SARS-CoV-2 sample lysis was performed on a 96well round-bottom plate. Briefly, $60 \mu \mathrm{L}$ of RNA lysis buffer was added to each well, followed by the addition of a $30 \mu \mathrm{L}$ sample. The plate was then placed on an orbital plate shaker for $5 \mathrm{~min}$ at $900 \mathrm{rpm}$ to lyse the sample. After lysis, $60 \mu \mathrm{L}$ of MagAttract beads/ethanol suspension (equivalent to $0.25 \mu \mathrm{L}$ bead stock) was added to the wells and then placed on the plate shaker again for $5 \mathrm{~min}$ at $900 \mathrm{rpm}$ for binding. (Note: the sample lysis and bead binding steps can also be performed in Eppendorf tubes or other vessels). The bead/sample mixture with a total volume of $150 \mu \mathrm{L}$ was transferred to the sample well of the OIL-TAS device to perform RNA extraction. The OIL-TAS device was then placed onto a custom magnetic extractor plate containing an array of neodymium magnets with a diameter of 0.0625 inches $(1.6 \mathrm{~mm})$ (Rare-Earth Disc Magnets, MAGCRAFT) positioned under each well, which causes the beads to coalesce above each magnet. The OIL-TAS device was then slid from right to left over the magnetic extractor to perform RNA extraction, which transports each magnetic bead cluster from the sample well through the two washing wells and into the detection well, a process that takes $<10$ s. The beads were then left in the detection well for isothermal amplification by placing the device in an incubator/oven set at $65^{\circ} \mathrm{C}$ for $35-50 \mathrm{~min}$. After amplification, beads were removed from the detection well (to allow for better colorimetric visualization) by placing the device on the magnetic extractor and sliding from left to right. Results of the colorimetric LAMP were then recorded by placing the device in a flatbed scanner (Epson Perfection V600 Photo with Epson Scan software). A positive reaction results in a color change of the droplet from pink to yellow, while a negative reaction droplet will remain pink.
Device operation (dual output device). Minor modifications to the operation protocol were made for the dual output OIL-TAS device (Fig. 6). The dual output OIL-TAS device was loaded with $50 \mu \mathrm{L}$ of silicone oil to each of the sample wells. Using a p20 pipette, $4 \mu \mathrm{L}$ of nuclease-free water, $50 \%$ ethanol, and LAMP master mix were sequentially added to washing well 2 , washing well 1 and the detection well, respectively, in the same manner as the single output device (except different primer sets were used in the two output detection wells). SARS-CoV-2 sample lysis and bead binding was performed in a 96-well deep well plate (or Eppendorf tubes). $120 \mu \mathrm{L}$ of RNA lysis buffer was added to each well of the well plate, followed by the addition of $60 \mu \mathrm{L}$ sample to the lysis buffer. The plate was then placed on an orbital plate shaker for $5 \mathrm{~min}$ at $900 \mathrm{rpm}$ to lyse the sample. After lysis, $90 \mu \mathrm{L}$ of MagAttract beads/ ethanol suspension (equivalent to $0.5 \mu \mathrm{L}$ bead stock) was added to the individual wells and then placed on the plate shaker again for $5 \mathrm{~min}$ at $900 \mathrm{rpm}$ for binding. The bead/ sample mixture with a total volume of $270 \mu \mathrm{L}$ was transferred to the sample well of the OIL-TAS device to perform RNA extraction. Magnetic extraction, amplification, and detection were performed as in the single output device.

Human clinical sample collection. Residual diagnostic nasopharyngeal (NP) swab specimens from routine testing were obtained from individuals who met COVID-19 clinical and/or epidemiological testing criteria. NP swabs were collected in various validated transport media (universal transport media (UTM), room temperature M4 (RT M4), phosphate-buffered saline (PBS), and saline). The activities in this study are determined to be not human subjects research by the University of Wisconsin - Madison Health Sciences Minimal Risk IRB (submission ID number: 2021-0594). All samples were de-identified (i.e., stripped of all 18 HIPAA identifiers using the "Safe Harbor" method in accordance with Sections $164.514(\mathrm{~b})(2)$ of the HIPAA privacy rule) prior to testing with OIL-TAS assays. Analyses of residual de-identified diagnostic specimens were performed under biosafety level- 2 containment. In order to enable safe handling for OIL-TAS assays, samples were heat-inactivated at $65^{\circ} \mathrm{C}$ for $30 \mathrm{~min}$ as previously described ${ }^{32}$ and then stored at $-80^{\circ} \mathrm{C}$ prior to running the assays. For real-time RTqPCR clinical assays, samples were directly tested without heat inactivation or freezing.

RT-qPCR assay. SARS-CoV-2 RNA was detected by RT-qPCR using either the Hologic Panther Fusion Emergency Use Authorized (EUA) SARS-CoV-2 Assay (Hologic, San Diego, CA) or a United States Centers for Disease Control and Prevention (CDC) multiplex EUA assay (UW Health) using Promega Maxwell Viral TNA reagents on the Promega Maxwell RSC48 and Promega MaxPrep instruments followed by amplifications run on the Roche LightCycler 480 real-time PCR instrument using Promega GoTaq ${ }^{\circledast}$ Probe qPCR Master mix (dUTP) and Promega GoScript ${ }^{\text {Ti }}$ RT Mix for 1-Step RT-qPCR. The Hologic Panther Fusion EUA and the CDC multiplex EUA (UW Health) assays target ORF1a/ORF1b and $N 1 / N 2$ viral sequences, respectively.

Quantification of carryover. Sample purification performance in the OIL-TAS process was evaluated by quantifying the amount of aqueous liquid carryover from one well to another following extraction. This was done by extracting magnetic beads through a fluorescent dye solution (acridine orange) as a model contaminant (Supplementary Fig. 2). In brief, a $4 \mu \mathrm{L}$ droplet of $500 \mu \mathrm{g} / \mathrm{mL}$ acridine orange solution in water was added to washing well 1 (input droplet) of the OIL-TAS device, and a $4 \mu \mathrm{L}$ droplet of deionized water was added to washing well 2 (output droplet). Carryover was defined by the amount of aqueous liquid that is carried over from the input droplet to the output droplet. In order to obtain a standard curve for fluorescence quantification, $4 \mu \mathrm{L}$ droplets of 2 -fold serially diluted acridine orange solution ranging from 10 to $0 \mu \mathrm{g} / \mathrm{mL}$ were added to the detection wells. $100 \mu \mathrm{L}$ of 1:400 diluted Qiagen MagAttract bead suspension in deionized water was added to the sample well, then extracted using the magnetic extractor through the acridine orange input droplets and into the water output droplets. The beads were subsequently removed from the output droplets by magnetic extraction in the opposite direction. The OIL-TAS device was then placed in a plate reader (PHERAstar FS, BMG Labtech), and the fluorescence intensity of the output droplets and standard curve droplets was measured with an excitation of $485 \mathrm{~nm}$ and emission of $520 \mathrm{~nm}$. The amount of carryover was determined by fitting the fluorescence intensity of the output droplet to the serially diluted acridine orange standard curve.

Data analysis and figure plotting. Data analysis and figure plotting was performed using Microsoft Excel, GraphPad Prism, and Roche LightCycler 480 software. Graphical illustrations were created with BioRender.com, Microsoft PowerPoint, Adobe Illustrator, and Blender (blender.org).

Reporting summary. Further information on research design is available in the Nature Research Reporting Summary linked to this article.

\section{Data availability}

The data supporting the findings of this study are available within the paper and its supplementary information files. Source data are provided with this paper. Any other relevant data are available from the authors upon reasonable request. Source data are provided with this paper. 
Received: 30 September 2020; Accepted: 10 June 2021;

Published online: 14 July 2021

\section{References}

1. Wang, H. et al. Phase-adjusted estimation of the number of Coronavirus Disease 2019 cases in Wuhan, China. Cell Discov. 6, 10 (2020).

2. Hui, D. S. et al. The continuing 2019-nCoV epidemic threat of novel coronaviruses to global health - the latest 2019 novel coronavirus outbreak in Wuhan, China. Int. J. Infect. Dis. 91, 264-266 (2020).

3. Moore, K. \& Conti, S. Grenova's plan to stop supply shortages in COVID-19 testing labs. Richmond Grid. https://richmondgrid.com/grenovas-plan-tostop-supply-shortages-in-covid-19-testing-labs/ (2020).

4. Wu, K. J. Coronavirus testing labs again lack key supplies. N. Y. Times https:// www.nytimes.com/2020/07/23/health/coronavirus-testing-supplyshortage. $\mathrm{html}(2020)$.

5. Li, C. et al. Exclusive liquid repellency: an open multi-liquid-phase technology for rare cell culture and single-cell processing. ACS Appl. Mater. Interfaces 10, 17065-17070 (2018).

6. Li, C., Niles, D. J., Juang, D. S., Lang, J. M. \& Beebe, D. J. Automated system for small-population single-particle processing enabled by exclusive liquid repellency. SLAS Technol. 24, 535-542 (2019).

7. Berry, S. M., Alarid, E. T. \& Beebe, D. J. One-step purification of nucleic acid for gene expression analysis via immiscible filtration assisted by surface tension (IFAST). Lab Chip 11, 1747-1753 (2011).

8. Berry, S. M., Strotman, L. N., Kueck, J. D., Alarid, E. T. \& Beebe, D. J. Purification of cell subpopulations via immiscible filtration assisted by surface tension (IFAST). Biomed. Microdevices 13, 1033-1042 (2011).

9. Berry, S. M., Regehr, K. J., Casavant, B. P. \& Beebe, D. J. Automated operation of immiscible filtration assisted by surface tension (IFAST) arrays for streamlined analyte isolation. J. Lab. Autom. 18, 206-211 (2013).

10. Casavant, B. P. et al. The VerIFAST: an integrated method for cell isolation and extracellular/intracellular staining. Lab Chip 13, 391-396 (2013).

11. Berry, S. M. et al. Using exclusion-based sample preparation (ESP) to reduce viral load assay cost. PLoS ONE 10, e0143631 (2015).

12. Juang, D. S., Berry, S. M., Li, C., Lang, J. M. \& Beebe, D. J. Centrifugationassisted immiscible fluid filtration for dual-bioanalyte extraction. Anal. Chem. 91, 11848-11855 (2019).

13. Notomi, T. et al. Loop-mediated isothermal amplification of DNA. Nucleic Acids Res. 28, E63 (2000).

14. Tanner, N. A., Zhang, Y. \& Evans, T. C. Jr Visual detection of isothermal nucleic acid amplification using pH-sensitive dyes. Biotechniques 58, 59-68 (2015).

15. Berry, S. M. et al. Weak protein-protein interactions revealed by immiscible filtration assisted by surface tension. Anal. Biochem. 447, 133-140 (2014)

16. Casavant, B. P., Guckenberger, D. J., Beebe, D. J. \& Berry, S. M. Efficient sample preparation from complex biological samples using a sliding lid for immobilized droplet extractions. Anal. Chem. 86, 6355-6362 (2014).

17. Berry, S. M. et al. AirJump: using interfaces to instantly perform simultaneous extractions. ACS Appl. Mater. Interfaces 8, 15040-15045 (2016).

18. MagNA Pure 96 Instrument. High-throughput Robotic Workstation for Fully Automated Purification of Nucleic Acids from up to 96 Samples. Roche Molecular Systems Inc. https://lifescience.roche.com/en_us/products/magnapure-96-instrument-382411-1.html\#overview (2020).

19. Applied Biosystems $s^{\text {in }}$ MagMAX ${ }^{\text {TM}}$-96 Viral RNA Isolation Kit. Thermo Fisher Scientific Inc., https://www.thermofisher.com/order/catalog/product/ AM1836\#/AM1836 (2017)

20. Magnetic Bead-based Isolation of Viral RNA/DNA and Bacterial DNANucleoMag Pathogen. Takara Bio Inc., https://www.takarabio.com/products/ nucleic-acid-purification/viral-dna-and-rna-purification-kits/nucleomagpathogen (2017)

21. Boom, R. et al. Rapid and simple method for purification of nucleic acids. $J$. Clin. Microbiol. 28, 495-503 (1990).

22. Escobar, M. D. \& Hunt, J. L. A cost-effective RNA extraction technique from animal cells and tissue using silica columns. J. Biol. Methods 4, e72 (2017).

23. He, H. et al. Integrated DNA and RNA extraction using magnetic beads from viral pathogens causing acute respiratory infections. Sci. Rep. 7, 45199 (2017).

24. Oberacker, P. et al. Bio-On-Magnetic-Beads (BOMB): open platform for highthroughput nucleic acid extraction and manipulation. PLoS Biol. 17, e3000107 (2019).

25. Yaffe, H. et al. LogSpin: a simple, economical and fast method for RNA isolation from infected or healthy plants and other eukaryotic tissues. BMC Res. Notes 5, 45 (2012).

26. Scallan, M. F. et al. Validation of a Lysis Buffer containing $4 \mathrm{M}$ guanidinium thiocyanate (GITC)/triton X-100 for extraction of SARS-CoV-2 RNA for
COVID-19 testing: comparison of formulated lysis buffers containing 4 to $6 \mathrm{M}$ GITC, Roche external lysis buffer and qiagen RTL lysis buffer. Preprint at bioRxiv https://doi.org/10.1101/2020.04.05.026435 (2020).

27. Broughton, J. P. et al. CRISPR-Cas12-based detection of SARS-CoV-2. Nat. Biotechnol. 38, 870-874 (2020).

28. Broughton, J. P. et al. A Protocol For Rapid Detection of the 2019 Novel Coronavirus SARS-CoV-2 Using CRISPR Diagnostics: SARS-CoV-2 DETECTR. Mammoth Biosciences Inc., (2020).

29. Color Genomics SARS-CoV-2 LAMP Diagnostic Assay Version 1.2. Color Genomics Inc., https://www.color.com/wp-content/uploads/2020/05/ColorLAMP-Diagnostic-Assay_v1-2_Updated-052120.pdf (2020).

30. Rabe, B. A. \& Cepko, C. SARS-CoV-2 detection using isothermal amplification and a rapid, inexpensive protocol for sample inactivation and purification. Proc. Natl. Acad. Sci. U. S. A. 117, 24450-24458 (2020).

31. Curtis, K. A. et al. A multiplexed RT-LAMP assay for detection of group M HIV-1 in plasma or whole blood. J. Virol. Methods 255, 91-97 (2018).

32. BEI Certificate of Analysis for product NR-52286 SARS-Related Coronavirus 2, Isolate USAWA1/2020, Heat Inactivated. BEI Resources, https://www. beiresources.org/Catalog/antigen/NR-52286.aspx (2020).

\section{Acknowledgements}

We would like to thank Dr. Brianna Mullins, Dr. Jay Warrick, and Dr. Scott Berry for helpful input and suggestions for the project. This study was funded by a UW WARF COVID-19 Accelerator Challenge award and the National Institutes of Health (NIH 1R01CA247479, NIH P30CA014520, NIH 1R01CA247479-01, NIH OD011106, NIH 5UL1TR002373-03).

\section{Author contributions}

D.S.J., T.D.J., D.M.D., and C.M.N. designed and performed experiments. M.A.A performed clinical sample preparation and RT-qPCR assays. D.J.B., D.H.O., T.C.F., and W. M.R. oversaw the study. D.S.J. and T.D.J. wrote the manuscript with input from all authors.

\section{Competing interests}

The authors declare the following competing interests. David J. Beebe holds equity in Bellbrook Labs LLC, Tasso Inc., Salus Discovery LLC, Lynx Biosciences Inc., Stacks to the Future LLC, Turba LLC, Onexio Biosystems LLC, and Flambeau Diagnostics LLC. David J. Beebe is also a consultant for Abbott Laboratories. Duane S. Juang holds equity in Turba LLC. A patent application (16/894273) of which D.J.B., D.S.J., T.D.J., D.H.O., and T.C.F. are inventor was filed through the Wisconsin Alumni Research Foundation for the OIL-TAS technology described in this work. All other authors declare no competing interests.

\section{Additional information}

Supplementary information The online version contains supplementary material available at https://doi.org/10.1038/s41467-021-24463-4.

Correspondence and requests for materials should be addressed to D.J.B.

Peer review information Nature Communications thanks Frederick Haselton and the other, anonymous, reviewer(s) for their contribution to the peer review of this work.

Reprints and permission information is available at http://www.nature.com/reprints

Publisher's note Springer Nature remains neutral with regard to jurisdictional claims in published maps and institutional affiliations.

\footnotetext{
Open Access This article is licensed under a Creative Commons Attribution 4.0 International License, which permits use, sharing, adaptation, distribution and reproduction in any medium or format, as long as you give appropriate credit to the original author(s) and the source, provide a link to the Creative Commons license, and indicate if changes were made. The images or other third party material in this article are included in the article's Creative Commons license, unless indicated otherwise in a credit line to the material. If material is not included in the article's Creative Commons license and your intended use is not permitted by statutory regulation or exceeds the permitted use, you will need to obtain permission directly from the copyright holder. To view a copy of this license, visit http://creativecommons.org/ licenses/by/4.0/.
}

(C) The Author(s) 2021 